\title{
Erratum
}

\section{Melamine and ammeline as nitrogen sources for turfgrasses}

In the above article, which was published in Fertilizer Research Volume 11, No. 1 (1987) pp. 79-86, Table 1 should have read as follows:

Table 1. Effect of $\mathrm{N}$ source on turf quality and average clipping yields

\begin{tabular}{lllll}
\hline Treatment & $\begin{array}{l}\text { Application } \\
\text { frequency }\end{array}$ & $\begin{array}{l}\text { Average } \\
\text { quality }\end{array}$ & $\begin{array}{l}\text { No. of dates of } \\
\text { unacceptable quality }\end{array}$ & $\begin{array}{l}\text { Average } \\
\text { yields }\end{array}$ \\
\hline & & & $--\%--$ & $-\mathrm{kg} \mathrm{ha}^{-1}-$ \\
& & & & \\
Urea & 4 & $8.4 \mathrm{a}^{\mathrm{b}}$ & 0 & $214 \mathrm{a}^{\mathrm{b}}$ \\
Urea/Melamine F & 2 & $6.4 \mathrm{bc}$ & 25 & $131 \mathrm{~b}$ \\
Melamine G & 2 & $6.8 \mathrm{~b}$ & 13 & $120 \mathrm{~b}$ \\
Melamine F & 2 & $4.3 \mathrm{~d}$ & 100 & $59 \mathrm{c}$ \\
Ammeline & 2 & $5.7 \mathrm{c}$ & 75 & $94 \mathrm{bc}$ \\
CV (\%) & - & 8.1 & - & 20.5 \\
& & $98 \mathrm{~kg} \mathrm{~N} \mathrm{ha}-1$ & & \\
Urea/Melamine F & 1 & $5.3 \mathrm{a}$ & 88 & $42 \mathrm{a}$ \\
Melamine G & 1 & $5.2 \mathrm{a}$ & 75 & $41 \mathrm{a}$ \\
Melamine F & 1 & $4.3 \mathrm{~b}$ & 100 & $63 \mathrm{a}$ \\
Ammeline & 1 & $5.0 \mathrm{a}$ & 75 & 28 \\
Control & - & 4.1 & 100 & 32.9 \\
CV (\%) & - & 5.5 & - & \\
\hline
\end{tabular}

average of eight dates for turf quality and seven for yields

$b$ Values within each $\mathrm{N}$ rate spanned by the same letter are not significantly different as tested by Duncan's New Multiple Range Test (5\%). Control plots were not included in analysis 